\title{
Fast ground-state cooling of mechanical resonators with time-dependent optical cavities
}

\author{
Yong Li, ${ }^{1,2}$ Lian-Ao Wu, ${ }^{3,4}$ and Z. D. Wang ${ }^{1}$ \\ ${ }^{1}$ Department of Physics and Center of Theoretical and Computational Physics, The University of Hong Kong, Pokfulam Road, \\ Hong Kong, China \\ ${ }^{2}$ Beijing Computational Science Research Center, Beijing 100084, China \\ ${ }^{3}$ Department of Theoretical Physics and History of Science, The Basque Country University (EHU/UPV), P.O. Box 644, \\ ES-48080 Bilbao, Spain \\ ${ }^{4}$ IKERBASQUE, Basque Foundation for Science, ES-48011 Bilbao, Spain
}

(Received 24 August 2010; published 6 April 2011)

\begin{abstract}
We propose a feasible scheme to cool down a mechanical resonator (MR) in a three-mirror cavity optomechanical system with controllable external optical driving fields. Under the Born-Oppenheimer approximation, the whole dynamics of the mechanical resonator and cavities is reduced to that of a time-dependent harmonic oscillator, whose effective frequency can be controlled through the optical driving fields. The fast cooling of the MR can be realized by controlling the amplitude of the optical driving fields. Significantly, we further show that the ground-state cooling may be achieved via the three-mirror cavity optomechanical system without the resolved sideband condition.
\end{abstract}

DOI: 10.1103/PhysRevA.83.043804

PACS number(s): 42.50.Wk, 03.65.-w, 42.50.Dv

\section{INTRODUCTION}

Ground-state cooling of nanomechanical resonators (MRs) has attracted great interest, as it is crucial in the improvement of detection precision of MRs [1,2], observation of quantum behaviors of macroscopic MRs [3], and quantum information processing based on MRs [4]. Over the years, methods to optimize the cooling of MRs in optomechanical systems [5-16] (or in electromechanical systems [17]) have been studied extensively. In these cooling schemes, the MR is coupled to a driven cavity through the optical radiation pressure and is cooled via the passive backaction cooling (also called self-cooling) [6,7,13-16] without a feedback loop. More significantly, the backaction ground-state cooling of MR [13-15], where the mean thermal occupation number of phonons $\bar{n}$ is less than 1 , can be achieved when the resolved sideband condition is satisfied.

Several experiments have reported progress in the backaction cooling of MR in optomechanical systems [5-11], in particular those approaching the ground states of MRs. Recent research shows that MRs can be cooled to states with a mean thermal occupation number $\bar{n}=35$ in an optical cavity [10], and $\bar{n}=3.8$ in a superconducting transmission line resonator [11].

Most existing models for ground-state cooling of MR in optomechanical systems use resolved sideband cooling. The thermal phonon number of the MR is reduced by the interaction between the MR and high-frequency auxiliary systems until the whole system eventually reaches equilibrium. Here we propose an alternative but efficient cooling method for MR in a three-mirror cavity (3MC) optomechanical system [18]. We notice that the MR and its auxiliary system, e.g., optical cavities, can be treated separately by the Born-Oppenheimer (BO) approximation [19]. The MR therefore behaves as a single-mode harmonic oscillator whose effective frequency is determined by the external optical driving fields. By controlling the amplitude of optical driving fields, we can quickly reduce the frequency of the bare MR, e.g., initially in a thermal equilibrium state, to a smaller effective frequency, but retaining the populations. In other words, the MR is cooled in a shorter time by doing work on the external system. Remarkably, by combining such a fast cooling scheme with another process, we find that it is feasible to achieve ground-state cooling of the MR with final effective frequencies that are the same as the bare one.

Our ground-state cooling mechanism is distinctly different from that of the conventional sideband cooling in optomechanical systems: (i) Realization of sideband cooling requires a long time until a steady state is reached. Here the MR may be cooled down to a nonsteady state at a short time and may later become hotter again because of interactions with environment. (ii) The optical drive is time independent in sideband cooling, while the present scheme needs a time-dependent and much stronger optical power. (iii) Sideband cooling of MR occurs when the optical detuning $\Delta$ approaches the bare frequency of MR, $\omega$, and the cooling condition here is far detuned from the cavity $(|\Delta| \gg \omega)$, which greatly simplifies the requirement for experimental control of the optical detuning. (iv) The decay rate of cavities in our scheme can be larger than the frequency of MR, while it is not allowed to be larger in resolved sideband cooling.

\section{THREE-MIRROR CAVITY CONFIGURATION}

Unlike the conventional two-mirror cavity (2MC) optomechanical system, we consider here an optomechanical system of 3MC configuration, as shown in Fig. 1. The mirror of MR with two perfectly reflecting surfaces is placed inside a cavity and the two other fixed mirrors are transmissive and subject to external optical driving fields. This setup is different from that in Ref. [8], where the MR is transmissive.

The Hamiltonian of the $3 \mathrm{MC}$ system can be written as

$$
H=H_{c}+H_{s},
$$




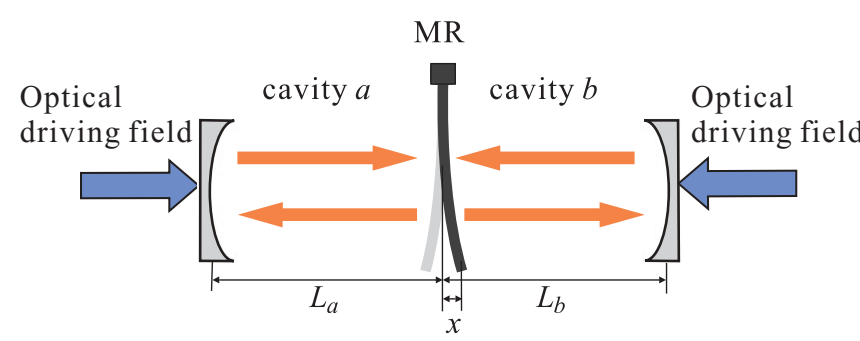

FIG. 1. (Color online) The schematics of the 3MC optomechanical system. The movable MR with two perfectly reflecting surfaces is placed inside a driven cavity with two transmissive fixed mirrors.

where $H_{s}=p^{2} / 2 m+m \omega^{2} x^{2} / 2$ is the Hamiltonian for a MR with effective mass $m$ and bare eigenfrequency $\omega$, and

$$
\begin{aligned}
H_{c}= & \hbar\left(\omega_{a}-G_{a} x\right) a^{\dagger} a+\hbar\left(\omega_{b}+G_{b} x\right) b^{\dagger} b \\
& +\hbar\left(\xi_{a} e^{i v_{a} t} a+\xi_{b} e^{i v_{b} t} b+\text { H.c. }\right)
\end{aligned}
$$

describes the optomechanical coupling between single-mode optically driven cavities $a$ and $b$ (with eigenfrequencies $\omega_{a}$ and $\omega_{b}$, respectively) and the MR. Here $\xi_{a, b}$ are the amplitudes of external optical driving fields to the cavities with corresponding drive frequencies $v_{a, b}, G_{a}=\omega_{a} / L_{a}$ and $G_{b}=\omega_{b} / L_{b}$ are the corresponding optomechanical coupling strengths via the radiation pressure, and $L_{a, b}$ are the lengths of cavities $a$ and $b$.

Similar to the case for electrons and nuclei in a molecule, the eigenfrequencies $\omega_{a}$ and $\omega_{b}$ of two cavities are much higher than the frequency $\omega$ of the MR such that the BO approximation can be employed to separate the degrees of freedom of MR from those of two cavities. For simplicity, but without loss of generality, we set $\omega_{a}=\omega_{b}, G_{a}=G_{b}=G$, $L_{a}=L_{b}=L, \xi_{a}=\xi_{a}^{*}=\xi_{b}=\xi_{b}^{*}=\xi$, and $v_{a}=v_{b}=v$. The cavity Hamiltonian can now be written as

$$
H_{c}=\hbar(\Delta-G x) a^{\dagger} a+\hbar(\Delta+G x) b^{\dagger} b+\hbar \xi(a+b+\text { H.c. })
$$

in a rotating frame with respect to $H_{f, 0}=\hbar v\left(a^{\dagger} a+b^{\dagger} b\right)$, where $\Delta \equiv\left(\omega_{a}-v\right)$ is the optical detuning at the absence of the motion of the MR.

We define operators $\widetilde{a}=a-\alpha$ and $\widetilde{b}=b-\beta$, where $\alpha=-\xi /(\Delta-G x)$ and $\beta=-\xi /(\Delta+G x)$, such that the Hamiltonian (3) can be expressed as

$$
H_{c}=\hbar(\Delta-G x) \widetilde{a}^{\dagger} \widetilde{a}+\hbar(\Delta+G x) \widetilde{b}^{\dagger} \widetilde{b}+c_{0}(x),
$$

where

$$
c_{0}(x)=\frac{-2 \hbar \Delta \xi^{2}}{\Delta^{2}-G^{2} x^{2}} \approx \frac{-2 \hbar \xi^{2}}{\Delta}\left(1+\frac{\omega^{2}}{\Delta^{2} L^{2}} x^{2}\right)
$$

is $x$ dependent. Note that we have used the condition $G x / \Delta \ll$ 1 and ignored the effects from the decay rates $\left(\kappa_{a}=\kappa_{b}=\kappa\right)$ of cavity fields based on the fact that $\kappa$ is much less than the detuning $\Delta$.

When the cavity fields are fixed for the state $|\widetilde{0}\rangle(\equiv|\widetilde{0}(x)\rangle)$ with $\widetilde{a}|\widetilde{0}\rangle=0$ and $\widetilde{b}|\widetilde{0}\rangle=0$, the effective Hamiltonian of the MR is

$$
\begin{aligned}
H_{\mathrm{eff}} & =\frac{\left(p+A_{\widetilde{0}}\right)^{2}}{2 m}+\frac{1}{2} m \omega^{2} x^{2}+c_{0}(x) \\
& \equiv \frac{p^{2}}{2 m}+\frac{1}{2} m \omega_{\mathrm{eff}}^{2} x^{2}+c_{r},
\end{aligned}
$$

where the effective frequency is given by $\omega_{\text {eff }}^{2}=\omega^{2}-$ $4 \hbar \xi^{2} \omega_{a}^{2} /\left(m \Delta^{3} L^{2}\right)$ and $c_{r}=-2 \hbar^{2} \xi^{2} / \Delta$ is a constant shift. We have assumed that the induced gauge potential $A_{\widetilde{0}}=$ $-i \hbar\langle\widetilde{0}|\nabla| \widetilde{0}\rangle=-i \hbar\left\langle\widetilde{0}(x)\left|\frac{\partial}{\partial x}\right| \widetilde{0}(x)\right\rangle=0$ if $|\widetilde{0}(x)\rangle$ is a real function. Then the MR behaves as a harmonic oscillator with the effective frequency determined by $\xi$ (equivalently the amplitudes of the optical driving fields), which is similar to the discussion according to the conventional optical springs [20].

The $3 \mathrm{MC}$ configuration distinguishes itself from the $2 \mathrm{MC}$ configuration because the high symmetry of the three-mirror cavity allows to significantly improve the trap stiffness and to partially remove the bistability, as addressed in Ref. [18]. The MR displacement in the effective Hamiltonian (5) is zero, while the corresponding MR displacement is finite in the $2 \mathrm{MC}$ configuration, making the dynamics of the $2 \mathrm{MC}$ more complicated.

\section{GENERAL FAST COOLING OF TIME-DEPENDENT MR}

Although the effective Hamiltonian (5) is obtained by using the time-independent $\mathrm{BO}$ approximation for the timeindependent $\xi$, a similar result also holds for a time-dependent parameter $\xi(t)=\xi_{0} f(t)$ when the factor $f(t) \quad(|f(t)|<1)$ varies very slowly in comparison with the detuning $\Delta$, i.e., $\dot{f}(t) / f(t) \ll \Delta$. In this case, the Schrödinger equation $H|\psi\rangle=i \hbar \partial_{t}|\psi\rangle$ can be simplified by a similar BO approximation with a highly nontrivial consideration of time dependency, as discussed in Ref. [21]. The BO approximation implies $\left\langle\widetilde{0}\left|H_{s}\right| \psi\right\rangle \approx H_{s}\langle\widetilde{0} \mid \psi\rangle$ such that $\langle\widetilde{0}|H| \psi\rangle \approx\left[H_{s}+\right.$ $\left.c_{0}(x)\right]\langle\widetilde{0} \mid \psi\rangle$. Here $\langle\widetilde{0} \mid \psi\rangle$ is the an instantaneous eigenwave function of the MR with the effective Hamiltonian $H_{c}, c_{0}(x)$ is the corresponding instantaneous eigenvalues and now is time dependent, $c_{0}(x)=c_{0}(x, t)$.

Based on the adiabatic theorem with conditions $\dot{f}(t) / f(t) \ll \Delta$ and the wave function $|\widetilde{0}\rangle$ being real, we obtain $\partial_{t}\langle\widetilde{0}| \approx 0$. The effective Schrödinger equation for the MR is then given by

$$
\left[H_{s}+c_{0}(x, t)\right]\langle\widetilde{0} \mid \psi\rangle=i \hbar \partial_{t}\langle\widetilde{0} \mid \psi\rangle .
$$

Therefore, the MR can be described as a time-dependent harmonic oscillator with the effective Hamiltonian

$$
H_{\text {eff }}(t)=H_{s}+c_{0}(x, t)=\frac{p^{2}}{2 m}+\frac{1}{2} m \omega_{\text {eff }}^{2}(t) x^{2} .
$$

Here the corresponding time-dependent eigenfrequency,

$$
\omega_{\mathrm{eff}}(t)=\omega \sqrt{1+\eta f^{2}(t)},
$$

with $\eta=-4 \hbar \xi_{0}^{2} \omega_{a}^{2} /\left(m \omega^{2} \Delta^{3} L^{2}\right)$, is controlled by the external optical field via the dimensionless function $f(t)$.

The dynamics of a time-dependent harmonic oscillator is analytically solvable using Lewis-Reisenfeld invariants [22]. Here we are only interested in the specific trajectories $\omega_{\text {eff }}(t)$ wherein the instantaneous populations at the initial time are the same as those at the final time, but the eigenfrequency and the corresponding average energy at the final time decreases. That means the harmonic oscillator has the same entropy at the initial and final times and is cooled by doing work 
on the external field, instead of having heat flowing out of it. Such a cooling trajectory does not depend on the initial state. The simplest way to realize the cooling trajectory is the adiabatic process where the populations remain the same all the time. The disadvantage is that the adiabatic process needs a long time, during which the relaxation of the harmonic oscillator may bring negative effects. Alternatively, an optimal bang-bang process has been proposed to achieve a cooling trajectory in a shorter time [23]. The effective frequency is real and stepwise function of time in this process, where the final instantaneous populations of the MR remain the same as the initial ones in terms of controlling the step values and durations of the effective frequency. Recently a fast optimal frictionless process $[24,25]$ was proposed to achieve atom cooling by choosing a certain trajectory of effective frequency, wherein the instantaneous effective frequency is allowed to be "imaginary."

Employing these methods, one is able to cool the MR in time-controllable optomechanical systems, as is done in atom cooling [23-25]. Let us estimate the feasibility of cooling a MR with reasonable empirical parameters: the bare eigenfrequency of MR $\omega / 2 \pi=134 \mathrm{kHz}$, the effective mass $m=50 \mathrm{pg}$ [8], the eigenfrequency of the optical cavity $\omega_{a} / 2 \pi \approx 7 \times 10^{14} \mathrm{~Hz}$, the optical detuning $\Delta / 2 \pi \approx 10^{7} \mathrm{~Hz}$, and the length of the optical cavity $L \approx 2 \mathrm{~mm}$. The optical driving fields are controllable and can have the time-dependent form $\xi=\xi_{0} f(t)$, with $\xi_{0} / 2 \pi \approx 10^{9} \mathrm{~Hz}$ and $|f(t)|<1$. With these parameters, we obtain $\eta \approx-9$. The effective frequency $\omega_{\text {eff }}(t)$ can be much smaller than the bare frequency $\omega$ of the MR, or even be "imaginary" by tuning $f(t)$.

Specifically, consider a MR in the thermal state with the mean phonon occupation number $\bar{n}\left(t_{\mathrm{i}}\right)=1 /\left[\exp \left(\hbar \omega / k_{B} T\right)-\right.$ $1] \approx k_{B} T / \hbar \omega(\gg 1)$ at the initial time $t_{\mathrm{i}}$, and $\omega_{\text {eff }}\left(t_{\mathrm{i}}\right)=\omega$. One can design a trajectory between $\omega_{\text {eff }}\left(t_{\mathrm{i}}\right)$ and the much lower effective frequency $\omega_{\text {eff }}\left(t_{\mathrm{f}}\right)$ at the final time [where $\omega_{\text {eff }}\left(t_{\mathrm{f}}\right) \equiv \omega / R$ with $R \gg 1$ and the corresponding $f\left(t_{\mathrm{f}}\right)=$ $\sqrt{\left(1-R^{-2}\right) / \eta}$ ] such that the final mean occupation number $\bar{n}\left(t_{\mathrm{f}}\right)=\bar{n}\left(t_{\mathrm{i}}\right)$. The energy of the MR is decreased by a factor $R$ after doing work on the external field, and the final effective temperature is reduced to $T_{\text {eff }}\left(t_{\mathrm{f}}\right) \equiv \hbar \bar{n}\left(t_{\mathrm{f}}\right) \omega_{\text {eff }}\left(t_{\mathrm{f}}\right) / k_{B}=$ $T / R \ll T$.

We have proposed a fast cooling mechanism for the MR as a time-dependent effective harmonic oscillator in a $3 \mathrm{MC}$ optomechanical system, where the final effective frequency of the MR is different from the initial one. However, the approach will not lead to the ground-state cooling since the final mean population number of the effective harmonic oscillator is the same as the original one which is usually much larger than 1 .

\section{GROUND-STATE COOLING OF MR}

In the preceding section, we obtain a fast cooling of MR wherein the final effective frequency of the MR is different from the initial one. People are mostly interested in the cooling of the MR with the final effective frequency of the MR being (nearly) equal to the original bare one, such as the sideband cooling of MR. In this section, we design a different cooling approach for the same 3MC optomechanical system to cool the MR close to its ground state in a short time, with the final effective frequency of the MR being equal to the original bare one.

We consider that the MR is originally (at $t=t_{\mathrm{o}}$ ) in a thermal equilibrium state $\rho\left(t_{0}\right)=e^{-H_{\text {eff }}\left(t_{0}\right) / k_{B} T} / \operatorname{Tr}\left(e^{-H_{\text {eff }}\left(t_{0}\right) / k_{B} T}\right)$ in a bath at a refrigeration temperature $T=20 \mathrm{mK}$ [26] in the absence of the optical driving fields. The corresponding mean occupation number of thermal phonons is $\bar{n}\left(t_{0}\right)=$ $1 /\left[\exp \left(\hbar \omega / k_{B} T\right)-1\right] \gg 1$. The MR is then subject to the optical radiation pressure of the driven cavities, described by the effective Hamiltonian in Eq. (7). We use the same preceding typical parameters, except with the optical detuning $\Delta / 2 \pi=-10^{7} \mathrm{~Hz}$ and with the maximum value of optical driving fields $\xi^{(0)} / 2 \pi \approx 10^{12} \mathrm{~Hz}$, and obtain $\eta \approx 9 \times 10^{6}$. At time $t=t_{\mathrm{i}}$ the MR evolves to a new thermal state $\rho\left(t_{\mathrm{i}}\right)=$ $e^{-H_{\text {eff }}\left(t_{\mathrm{i}}\right) / k_{B} T} / \operatorname{Tr}\left(e^{-H_{\text {eff }}\left(t_{\mathrm{i}}\right) / k_{B} T}\right)$, with a mean thermal occupation number $\bar{n}\left(t_{\mathrm{i}}\right)=1 /\left\{\exp \left[\hbar \omega_{\mathrm{eff}}\left(t_{\mathrm{i}}\right) / k_{B} T\right]-1\right\}$. We take $f\left(t_{\mathrm{i}}\right)=$ 1 such that $\omega_{\text {eff }}\left(t_{\mathrm{i}}\right) \approx 3000 \omega$ and $\bar{n}\left(t_{\mathrm{i}}\right) \approx 0.66<1 \ll \bar{n}\left(t_{\mathrm{o}}\right) \approx$ 3200. The intermediate trajectory between $t_{\mathrm{o}}$ and $t_{\mathrm{i}}$ may be arbitrary, on the condition that the MR is in the thermal state at time $t_{\mathrm{i}}$.

We need to design a special trajectory of the effective frequency $\omega_{\mathrm{eff}}(t)$ from $t=t_{\mathrm{i}}$ to the final time $t=t_{\mathrm{f}}$ such that $\omega_{\mathrm{eff}}\left(t_{\mathrm{f}}\right)$ is equal to the original bare frequency $\omega$ and the corresponding state $\rho\left(t_{\mathrm{f}}\right)=\rho\left(t_{\mathrm{i}}\right)$. Therefore, the final mean thermal occupation number $\bar{n}\left(t_{\mathrm{f}}\right)=\bar{n}\left(t_{\mathrm{i}}\right) \approx 0.66$, which indicates that the MR is cooled near its ground state.

We now focus on designing such a trajectory of $\omega_{\text {eff }}(t)$ in terms of the Lewis-Riesenfeld invariant of motion [22]. The invariant of motion for our MR harmonic oscillator is $I(t)=$ $m \omega_{0}^{2} x^{2} /\left[2 b^{2}(t)\right]+[b(t) p-m \dot{b}(t) x]^{2} / 2$, where $\omega_{0}=\omega_{\mathrm{eff}}\left(t_{\mathrm{i}}\right)$. The dimensional real function $b(t)$ satisfies the condition $\ddot{b}(t)+\omega_{\text {eff }}^{2}(t) b=\omega_{0}^{2} / b^{3}(t)$ [22], which is used in Ref. [24] for cold atoms. We show that the simplest polynomial choice of $b(t)$ [27] will lead to an above-required cooling trajectory, which can be realized by controlling the parameters $f(t)$, as shown by the solid and dashed lines in Fig. 2.

In addition, the cooling process could even be faster if imaginary frequencies $\omega_{\mathrm{eff}}(t)$ could be allowed as done in Ref. [24], where the imaginary frequencies cooling scheme were first proposed. The single optical mode Hamiltonian (4) fails to provide such imaginary values of $\omega_{\text {eff }}(t)$ since $\eta$ is positive for the fixed parameters above. For that purpose, we could adopt two optical modes in each cavity for the similar symmetric 3MC configuration. A self-adjoint Hamiltonian for the model with two optical modes in each cavity in a rotating frame should be

$$
\begin{aligned}
H_{c}^{\prime}= & \hbar\left(\Delta_{1}-G_{1} x\right) a_{1}^{\dagger} a_{1}+\hbar\left(\Delta_{1}+G_{1} x\right) b_{1}^{\dagger} b_{1} \\
& +\hbar\left(\Delta_{2}-G_{2} x\right) a_{2}^{\dagger} a_{2}+\hbar\left(\Delta_{2}+G_{2} x\right) b_{2}^{\dagger} b_{2} \\
& +\hbar\left[\xi_{1}\left(a_{1}+b_{1}\right)+\xi_{2}\left(a_{2}+b_{2}\right)+\text { H.c. }\right]
\end{aligned}
$$

where $a_{1,2}\left(b_{1,2}\right)$ denote the annihilation operators for the two modes in cavity $a$ (cavity $b$ ) with frequencies $\omega_{1,2}$. The coupling strengths of optical radiation pressure are $G_{1,2}=$ $\omega_{1,2} / L . \quad \xi_{1}:=\xi_{1}^{(0)} f_{1}(t)$ and $\xi_{2}:=\xi_{2}^{(0)} f_{2}(t) \quad\left(\left|f_{1,2}(t)\right|<1\right)$ are the amplitudes of the external time-dependent optical 

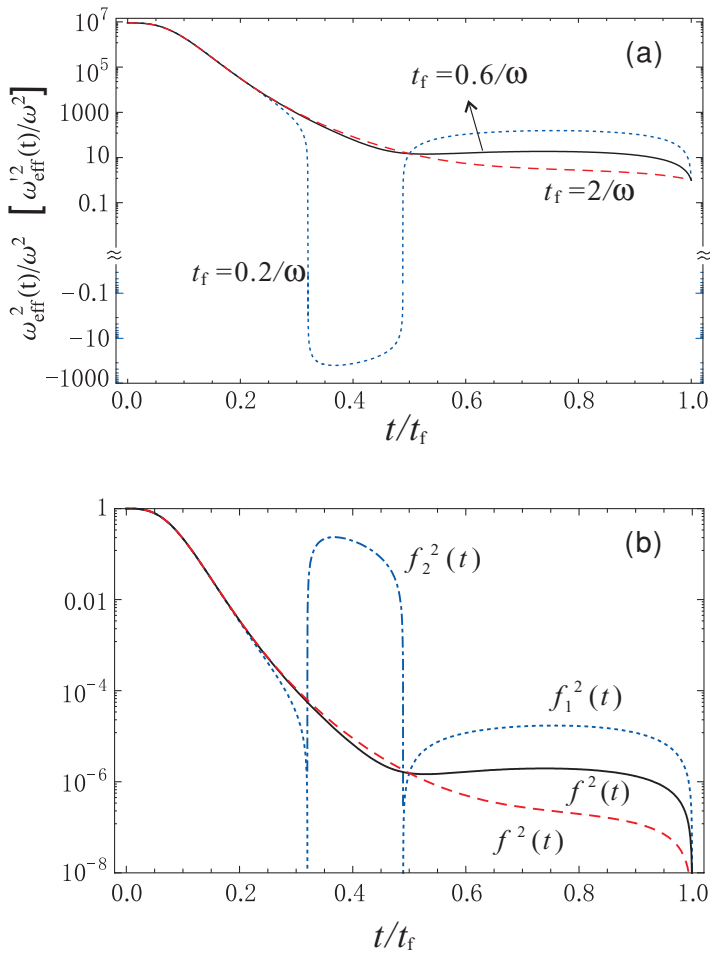

FIG. 2. (Color online) (a) The square of instantaneous eigenfrequency $\omega_{\text {eff }}^{\prime}(t)$ [or $\omega_{\text {eff }}(t)$ ] of MR from $\omega_{\text {eff }}^{\prime}\left(t_{\mathrm{i}}=0\right)=3000 \omega$ to $\omega_{\text {eff }}^{\prime}\left(t_{\mathrm{f}}\right)=\omega$ for different durations: $t_{\mathrm{f}}=0.2 / \omega$ (blue dotted line); $t_{\mathrm{f}}=0.6 / \omega$ (black solid line); $t_{\mathrm{f}}=2 / \omega$ (red dashed line). Here, the bare frequency of MR is $\omega=2 \pi \times 134 \mathrm{kHz}$. The negative value is due to an imaginary $\omega_{\text {eff }}^{\prime}(t)$, which appears for the case of short evolution time $t_{\mathrm{f}}$. (b) The corresponding time-dependent control parameters $f^{2}(t)\left[f_{1}^{2}(t)\right]$ (proportional to the optical powers) and $f_{2}^{2}(t)$ (the blue dotted-dashed line, which contributes mainly to the "imaginary" $\left.\omega_{\mathrm{eff}}^{\prime}(t)\right)$.

driving fields with frequencies $v_{1,2}$. The optical detunings are $\Delta_{1} \equiv\left(\omega_{1}-v_{1}\right)$ and $\Delta_{2} \equiv\left(\omega_{2}-v_{2}\right)$.

Following the preceding discussion, the effective timedependent Hamiltonian for the MR becomes

$$
H_{\mathrm{eff}}^{\prime}(t) \equiv \frac{p^{2}}{2 m}+\frac{1}{2} m \omega_{\mathrm{eff}}^{\prime 2}(t) x^{2},
$$

where the time-dependent eigenfrequency is given by

$$
\omega_{\mathrm{eff}}^{\prime 2}(t)=\omega^{2}\left[1+\eta_{1} f_{1}^{2}(t)+\eta_{2} f_{2}^{2}(t)\right]
$$

with two coefficients $\eta_{1}=-4 \hbar \xi_{1}^{(0) 2} \omega_{1}^{2} /\left(m \omega^{2} \Delta_{1}^{3} L^{2}\right)$ and $\eta_{2}=$ $-4 \hbar \xi_{2}^{(0) 2} \omega_{2}^{2} /\left(m \omega^{2} \Delta_{2}^{3} L^{2}\right)$.

We now have more effective control over the timedependent eigenfrequency $\omega_{\text {eff }}^{\prime}(t)$ through two independent parameters $f_{1,2}(t)$ (proportional to the external input optical powers) and will use the same typical parameters for both modes as before, except for the optical frequencies $\omega_{1} / 2 \pi \approx 7 \times 10^{14}$ $\mathrm{Hz}, \omega_{2} / 2 \pi \approx 6 \times 10^{14} \mathrm{~Hz}$, the maximum amplitude of optical driving fields $\xi_{1}^{(0)} / 2 \pi \approx 10^{12} \mathrm{~Hz}, \xi_{2}^{(0)} / 2 \pi \approx 10^{10} \mathrm{~Hz}$, and the detunings $\Delta_{1} / 2 \pi=-10^{7} \mathrm{~Hz}$ and $\Delta_{2} / 2 \pi=10^{7} \mathrm{~Hz}$. Therefore, the coefficients $\eta_{1} \approx 9 \times 10^{6}$ and $\eta_{2} \approx-670$. Thus, the effective frequency could be imaginary in the process when $f_{2}(t)$ is much larger than $f_{1}(t)$, and the cooling can be achieved even faster (see the blue dotted and dashed-dotted lines in Fig. 2).

We wish to mention that our ground-state cooling scheme requires a strong input optical power to obtain a strong modulation for the effective frequency. The maximum value of the optical input power is $P=\hbar \omega_{a} \xi^{(0) 2} / 2 \kappa \approx 1 \mathrm{~W}$ for the maximum $\xi^{(0)} / 2 \pi \approx 10^{12} \mathrm{~Hz}$, where the decay rate of the optical modes of the cavities is assumed as $\kappa / 2 \pi \approx 10^{6} \mathrm{~Hz}$. The corresponding high finesse cavities, with $F=\pi c /(2 L \kappa) \approx$ $3.8 \times 10^{4}$, are required. We remark that the adiabatic condition for the time-dependent BO approximation, $\dot{f}(t) / f(t) \ll|\Delta|$ and $\dot{f}_{1,2}(t) / f_{1,2}(t) \ll\left|\Delta_{1,2}\right|$, is satisfied for the trajectories in Fig. 2.

Our general analysis shows that we can realize groundstate cooling for a MR with the present approach. Even if the coupling between the MR and the optical cavity is removed by moving the optical driving fields away from the fixed mirrors, the MR will remain in the same ground state for a longer time after $t_{\mathrm{f}}$, until it approaches a new thermal equilibrium with its bath. During this period of time, one can perform quantum operations, such as quantum information processing, on the MR. Note that the controlled trajectory between $\omega_{\text {eff }}\left(t_{\mathrm{i}}\right)$ and $\omega_{\text {eff }}\left(t_{\mathrm{f}}\right)$ is preformed in a short time of a fraction of $1 / \omega$, such that the relaxation process of the MR with a high $Q$ factor (e.g., 10 $0^{5}$ ) can be ignored.

\section{CONCLUSIONS}

We propose a feasible scheme to cool a MR through a $3 \mathrm{MC}$ optomechanical system. In the limit of large optical detuning, we show that the optical fields can be eliminated and the degree of freedom of the MR in the general Hamiltonian can be described by a time-dependent harmonic oscillator. By controlling the amplitude of the external input optical driving fields, we can manipulate the time-dependent effective frequency and obtain the cooling of the MR via keeping the same populations of instantaneous levels at the initial and final times during a special trajectory of short time period. The ground-state cooling of MR can be realized as well. It is encouraging that a similar trajectory has been implemented experimentally [25] for atom cooling. We believe that the same technique is applicable to our scheme for a mechanical resonator.

\section{ACKNOWLEDGMENTS}

We would like to thank Dr. P. Zhang for helpful discussions. This work was supported by the RGC grant of Hong Kong, No. HKU7044/08P, the Ikerbasque Foundation Start-up, the Basque Government (Grant No. IT472-10), the Spanish MEC No. FIS2009-12773-C02-02, and the State Key Program for Basic Research of China (No. 2006CB921800). 
[1] V. B. Braginsky and A. B. Manukin, Measurement of Weak Forces in Physics Experiments (The University of Chicago Press, Chicago, 1977).

[2] C. M. Caves et al., Rev. Mod. Phys. 52, 341 (1980); M. F. Bocko et al., ibid. 68, 755 (1996); M. D. LaHaye et al., Science 304, 74 (2004); B. Abbott et al., Phys. Rev. Lett. 95, 221101 (2005) .

[3] S. Mancini, V. Giovannetti, D. Vitali, and P. Tombesi, Phys. Rev. Lett. 88, 120401 (2002); W. Marshall, C. Simon, R. Penrose, and D. Bouwmeester, ibid. 91, 130401 (2003); L. F. Wei, Y. X. Liu, C. P. Sun, and F. Nori, ibid. 97, 237201 (2006); F. Xue, L. Zhong, Y. Li, and C. P. Sun, Phys. Rev. B 75, 033407 (2007).

[4] S. Mancini, D. Vitali, and P. Tombesi, Phys. Rev. Lett. 90, 137901 (2003); K. Hammerer, M. Aspelmeyer, E. S. Polzik, and P. Zoller, ibid. 102, 020501 (2009); L. Tian and P. Zoller, ibid. 93, 266403 (2004).

[5] C. H. Metzger and K. Karrai, Nature (London) 432, 1002 (2004).

[6] S. Gigan et al., Nature (London) 444, 67 (2006).

[7] O. Arcizet et al., Nature (London) 444, 71 (2006).

[8] J. D. Thompson et al., Nature (London) 452, 72 (2008); J. D. Thompson Sankey et al., Nat. Phys. 6, 707 (2010).

[9] A. Schliesser et al., Nat. Phys. 4, 415 (2008).

[10] S. Groblacher et al., Nat. Phys. 5, 485 (2009).

[11] T. Rocheleau et al., Nature (London) 463, 72 (2010).

[12] S. Mancini, D. Vitali, and P. Tombesi, Phys. Rev. Lett. 80, 688 (1998).

[13] I. Wilson-Rae, N. Nooshi, W. Zwerger, and T. J. Kippenberg, Phys. Rev. Lett. 99, 093901 (2007).

[14] F. Marquardt, J. P. Chen, A. A. Clerk, and S. M. Girvin, Phys. Rev. Lett. 99, 093902 (2007).

[15] Y. Li, Y. D. Wang, F. Xue, and C. Bruder, Phys. Rev. B 78, 134301 (2008); Y. Li, Z. D. Wang, and C. P. Sun, Eur. Phys. J. D 61, 215 (2011).

[16] T. J. Kippenberg et al., Opt. Express 15, 17172 (2007).

[17] L. Tian and P. Zoller, Phys. Rev. Lett. 93, 266403 (2004); P. Zhang, Y. D. Wang, and C.P. Sun, ibid. 95, 097204 (2005);
A. Naik et al., Nature (London) 443, 193 (2006); Y.-D. Wang, Y. Li, F. Xue, C. Bruder, and K. Semba, Phys. Rev. B 80, 144508 (2009); K. Jacobs, H. I. Nurdin, F. W. Strauch, and M. James, e-print arXiv:1003.2653.

[18] M. Bhattacharya and P. Meystre, Phys. Rev. Lett. 99, 073601 (2007); P. Meystre et al., J. Opt. Soc. Am. B 2, 1830 (1985).

[19] M. Born and J. R. Oppenheimer, Ann. Phys. 84, 457 (1927).

[20] B. S. Sheard, M. B. Gray, C. M. Mow-Lowry, D. E. McClelland, and S. E. Whitcomb, Phys. Rev. A 69, 051801(R) (2004), and references therein.

[21] T. J. Gil, S. Shi, A. Askar, and H. A. Rabitz, Phys. Rev. A 45, 6479 (1992).

[22] H. R. Lewis et al., J. Math. Phys. (NY) 10, 1458 (1969); M. V. Berry et al., J. Phys. A 17, 1805 (1984); V. V. Dodonov et al., J. Math. Phys. (NY) 34, 3391 (1993); M. A. Lohe, J. Phys. A 42, 035307 (2009).

[23] P. Salamon et al., Phys. Chem. Chem. Phys. 11, 1027 (2009).

[24] X. Chen, A. Ruschhaupt, S. Schmidt, A. delCampo, D. GueryOdelin, and J.G. Muga, Phys. Rev. Lett. 104, 063002 (2010); J. G. Muga et al., J. Phys. B 43, 1 (2010).

[25] J.-F. Schaff, X. L. Song, P. Vignolo, and G. Labeyrie, Phys. Rev. A 82, 033430 (2010)

[26] We notice that the conditions of initial temperature $20 \mathrm{mK}$ and the strong optical input power $1 \mathrm{~W}$ may be at or beyond the borderline of current optomechanical experiments. We choose such ideal parameters to show how the ground-state cooling of the MR with bare frequency $\omega=2 \pi \times 134 \mathrm{kHz}$ can be reached. For the MRs with larger bare frequency and smaller effective masses, less laser power and a higher initial temperature are required to achieve its ground-state cooling.

[27] That is, $\quad b(t)=6 \zeta\left[t /\left(t_{\mathrm{f}}-t_{\mathrm{i}}\right)\right]^{5}-15 \zeta\left[t /\left(t_{\mathrm{f}}-t_{\mathrm{i}}\right)\right]^{4}+$ $10 \zeta\left[t /\left(t_{\mathrm{f}}-t_{\mathrm{i}}\right)\right]^{3}+1$, with $\zeta \equiv \sqrt{\omega_{\mathrm{eff}}\left(t_{\mathrm{i}}\right) / \omega_{\mathrm{eff}}\left(t_{\mathrm{f}}\right)}-1$. 